\title{
A step towards risk-based decision support for ships - Evaluation of limit states using parallel system analysis
}

Nielsen, Ulrik Dam; Friis-Hansen, Peter; Jensen, Jørgen Juncher

Published in:

Marine Structures

Link to article, DOI:

10.1016/j.marstruc.2008.08.002

Publication date:

2009

Document Version

Early version, also known as pre-print

Link back to DTU Orbit

Citation (APA):

Nielsen, U. D., Friis-Hansen, P., \& Jensen, J. J. (2009). A step towards risk-based decision support for ships Evaluation of limit states using parallel system analysis. Marine Structures, 22(2), 209-224.

https://doi.org/10.1016/j.marstruc.2008.08.002

\section{General rights}

Copyright and moral rights for the publications made accessible in the public portal are retained by the authors and/or other copyright owners and it is a condition of accessing publications that users recognise and abide by the legal requirements associated with these rights.

- Users may download and print one copy of any publication from the public portal for the purpose of private study or research.

- You may not further distribute the material or use it for any profit-making activity or commercial gain

- You may freely distribute the URL identifying the publication in the public portal 


\title{
A Step towards Risk-based Decision Support for Ships - Evaluation of Limit States using Parallel System Analysis
}

\author{
Ulrik Dam Nielsen*, Peter Friss-Hansen and Jørgen Juncher Jensen \\ Department of Mechanical Engineering, \\ Technical University of Denmark, DK-2800 Lyngby, Denmark
}

\begin{abstract}
Onboard decision support systems (DSS) are used to increase the operational safety of ships. Ideally, DSS can estimate future ship responses within on time scale of the order of 1-3 hours taking into account speed and course changes, assuming stationary sea states. In principle, the calculations depend on a large amount of operational and environmental parameters, which will be known only in the statistical sense. The present paper suggests a procedure to incorporate random variables and associated uncertainties in the calculations of the outcrossing rates that are the basis for risk-based DSS. The procedure is based on parallel system analysis, and the paper derives and describes the main ideas. The concept is illustrated by an example, where the limit state of a non-linear ship response is considered. The results from the parallel system analysis are in agreement with corresponding Monte Carlo simulations. However, the computational speed of the parallel system analysis proved slower than expected.
\end{abstract}

Key words: Risk-based decision support for ships, parallel system analysis, non-linear ship responses, uncertainty modelling, mean outcrossing rate, importance factors

\section{InTRODUCTION}

In the future, various kinds of onboard guidance and monitoring systems will likely become standard practice in ships to assist the ship master navigate under non-normal conditions; in particular during storm and rough sea. In principle, this means that an objective system - the decision support system (DSS) - supports the ship master in making suitable decisions with respect to speed and course changes, so that specific ship responses are kept at an acceptable level.

There exists a variety of decision support systems of which many are already in operational use. A comprehensive overview of state-of-the-art systems has been carried out within the EU project ADOPT (Advanced Decision Support System for Ship Design, Operation and Training), e.g. Tellkamp et al. [21], under the Sixth Framework Programme governed by the European Community. Some of the systems that are considered include, among others, the OCTOPUS Onboard [18] by Amarcon, the Seaware EnRoute Live [19] by Seaware Onboard, the Vessel Optimization and Safety System [22] by Ocean Systems, and the SeaSense system [15] by FORCE technology. The authors have themselves been involved in parts of the development of the SeaSense system. This system

\footnotetext{
*Corresponding author. E-mail: udn@mek.dtu.dk Tel.: +4545251970
} 
fuses the information of several onboard sensors which measure responses such as vertical wave induced bending moment amidship, accelerations at AP and FP, relative wave height, and green water occurrences on deck. The SeaSense system makes estimations of the sea state by use of a simplified version of the wave buoy-analogy, where measured ship responses are used to estimate the directional wave spectrum, e.g. Nielsen [16] and Iseki and Terada [6].

In general, decision support systems combine information on the on-site sea state with various kinds of pre-calculated or online response calculations to obtain statistical information about future responses to be expected. Implicitly, the statistical predictions depend on all operational parameters such as speed, metacentric height, relative wave heading, mass distribution, etc. In addition, the predictions will be directly influenced by parameters describing the sea state; e.g. significant wave height and zero-upcrossing period. Under real operational conditions the problem is that none of these parameters are known exactly, which means that the parameters must be described in terms of random variables with related uncertainties. If guidance systems are to give the most valuable and/or reliable support, it is therefore essential that all the uncertainties in the random variables are taken into account. This area has therefore been given special attention by the ADOPT project and, thus, one of the work packages (WP3) specifically focused on strategies for limit state evaluation including uncertainty analysis and the present paper outlines some of the findings of WP3 with special consideration to parallel system analysis for obtaining the mean outcrossing rate. Some general ideas and concepts of risk-based decision support systems are given in Bitner-Gregersen and Skjong [1], and Spanos et al. [20] considers the concept of a DSS to handle, specifically, Gaussian, narrow-banded processes.

It should be emphasised that the focus of the paper, as it will appear, basically concerns the calculation of the expected value of the mean outcrossing rate of a given process. Although the presented methodology here has its overall relevance to decision support systems for ships, as cited in the title of the paper, these systems and their principles and applications will not be dealt with in any further details. This means e.g. that the central role of a DSS - the interaction of predictions with measurements from sensors - is not touched upon in the paper.

\section{Definitions and Objective}

Figure 2.1 illustrates the behaviour in time $t$ of a general response $Z(t, \mathbf{X})$ that depends, in some way, on the parameters of the vector $\mathbf{X}$. It is assumed that the response depends non-linearly on the wave excitation. In the figure, a threshold value, say $\zeta$, has been indicated by a dashed horizontal line. Thus, the number of crossings relative to the threshold value can be counted, which means that the outcrossing rate for the specific period of time can be obtained. Obviously, it would be possible to obtain an estimate for the mean outcrossing rate $\bar{\nu}$ of the process $Z(t, \mathbf{X})$ if a sufficiently long time span were considered.

The expected loss $L_{i}(T)$ of some unwanted event $i$ within time $T$ is defined by

$$
L_{i}(T)=\bar{\nu}_{i} \mu_{i} T
$$


where $\bar{\nu}_{i}$ is the expected probability of failure per time unit, i.e. the expected value of the mean outcrossing rate, and $\mu_{i}$ is the consequence/cost of failure that is materialised when the event occurs. The general idea of decision support systems is thus to supply guidance, so that the expected loss is minimised for the required ship operation; in this context, that would be the traveling between point $\mathrm{A}$ and $\mathrm{B}$ with appropriate choices of speed and course relative to the encountered wave system. Hence, the decision support system must, by some means, simulate the process $Z(t, \mathbf{X})$, so that the mean outcrossing rate is obtained for a set of alternative speeds and courses. It is noteworthy that a decision support system shall be able to handle, simultaneously, a number of events with associated consequences. A DSS therefore needs to supply guidance in respect of the total loss

$$
L(T)=\sum_{i \in \text { all events }} L_{i}(T)=\sum_{i \in \text { all events }} \bar{\nu}_{i} \mu_{i} T
$$

The objective of this paper is to calculate the expected value of the mean outcrossing rate $\bar{\nu}=$ $E_{X}\left[\nu^{+}(\mathbf{X})\right]$ taking into account all uncertainties of the associated random variables, $\mathbf{X}$, e.g. vessel speed, relative wave heading, instantaneous righting arm, etc. In the terminology of reliability theory, the expectation of the mean outcrossing rate is obtained by (successive) use of parallel system analysis. However, the use of parallel system analysis is not the only procedure that can be applied to establish the expected value of the mean outcrossing rate, as the value can also be obtained by brute force time series simulations applying e.g. Monte Carlo simulation. Thus, similar realisations to that in Figure 2.1 can be used to count the number of outcrossings. This study will focus primarily on the parallel system analysis based on FORM/SORM, but the study is not to favour this procedure compared to a procedure based on brute force simulations. Although FORM/SORM in general performs faster for continuous distributions of the random variables, a general comparative study is difficult to make since the two procedures are complementary methods. The parallel system analysis by use of FORM/SORM and brute force simulation have their own advantages and disadvantages as shown in Table 2.1.

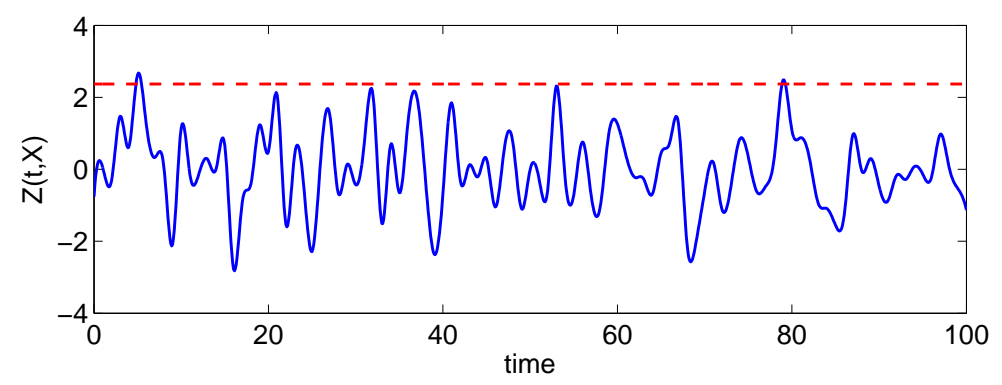

FiguRE 2.1. Time variation of general (non-linear) process. 
TABLE 2.1. Advantages and disadvantages of parallel system analysis by use of FORM/SORM and brute force simulation for DSS for ship operation.

\begin{tabular}{|c|c|c|}
\hline & FORM/SORM & Brute force simulations \\
\hline Advantages & $\begin{array}{l}\text { - Calculation time is independent of } \\
\text { probability level } \\
\text { - The design point is a by-product of } \\
\text { the analysis (i.e. the wave pattern } \\
\text { can be obtained) } \\
\text { - Sensitivity factors are a by-product } \\
\text { of the analysis }\end{array}$ & $\begin{array}{l}\text { - Any limit state may be calculated } \\
\text { - Multiple limit states may be cal- } \\
\text { culated at the same time at limited } \\
\text { cost; the ship motion analysis is } \\
\text { the hard part } \\
\text { - Outcrossing rates may be esta- } \\
\text { blished for several levels }\end{array}$ \\
\hline Disadvantages & $\begin{array}{l}\text { - Each new limit state requires a new } \\
\text { analysis } \\
\text { - Each new level requires a new } \\
\text { analysis } \\
\text { - Singular limit state functions may } \\
\text { behave badly, that is, a less } \\
\text { efficient search algorithm needs to } \\
\text { be applied }\end{array}$ & $\begin{array}{l}\text { - Rare events are difficult (time } \\
\text { consuming) to obtain } \\
\text { - Difficult to extract directly what } \\
\text { drives the conditions at failure (e.g. } \\
\text { the wave pattern) } \\
\text { - Cost expensive to calculate sensi- } \\
\text { tivity factors }\end{array}$ \\
\hline
\end{tabular}

The use of FORM (or SORM) requires the set-up of a limit state function $g(Z(t, \mathbf{X}), \zeta)$ and, in general, a limit state can be defined by

$$
g(Z(t, \mathbf{X}), \zeta)=\left\{\begin{array}{rc}
<0 ; & \text { unsafe domain } \\
=0 ; & \text { failure surface } \\
>0 ; & \text { safe domain }
\end{array}\right.
$$

With attention drawn to Figure 2.1, a limit state function may be given by

$$
g \equiv \zeta-Z(t, \mathbf{X})
$$

The parallel system approach - based on FORM (or SORM) - should be applied only to processes that are non-linear or non-Gaussian with respect to the wave excitations. The reason is that closedform expressions can be derived for the mean outcrossing rate if a linear and Gaussian process is considered and, hence, the computational time is significantly reduced. Closed-form expressions can not be established for the outcrossing rate of non-linear processes, which leads to a solution based on parallel system analysis. In this study, a limit state depending on the wave induced acceleration will be established and studied.

\section{Limit State Formulation}

This paper addresses a strategy to obtain the expected value of the mean outcrossing rate of a given non-linear process. The actual kind of process is of minor importance, but for illustrative and conceptual purposes, the limit state formulation concerns the wave induced acceleration and, 
in general, several limit states relevant to decision support systems will be dependent on the wave induced acceleration of the ship. Examples of related limit states are: comfort level for passengers and crew, sliding of unlashed trucks, break of lashing systems for containers and/or lashed trucks.

3.1. Wave Induced Acceleration. In six degrees of freedom the ship motions are surge, sway, heave, roll, pitch, and yaw, $\boldsymbol{\eta}=\left[\eta_{1}, \eta_{2}, \eta_{3}, \eta_{4}, \eta_{5}, \eta_{6}\right]^{T}$, relative to the centre of gravity (COG) of the ship. Thus, with the fixed ship coordinate system located at COG, the $y$ - and the $z$-component of the wave induced acceleration at the point $\mathbf{x}_{0}=\left(x_{0}, y_{0}, z_{0}\right)$ are given as

$$
\begin{aligned}
& a_{y}=\ddot{\eta}_{2}-\ddot{\eta}_{4} z_{0}+\ddot{\eta}_{6} x_{0} \\
& a_{z}=\ddot{\eta}_{3}+\ddot{\eta}_{4} y_{0}-\ddot{\eta}_{5} x_{0}
\end{aligned}
$$

where a superscript dot denotes differentiation with respect to time. It should be noted that only rigid body motions are dealt with.

With respect to the maximum acceleration, contributions come mainly from heave, pitch and roll, so that sway and yaw may be omitted. Hence,

$$
\begin{gathered}
a_{y}=-\ddot{\eta}_{4} z_{0} \\
a_{z}=\ddot{\eta}_{3}+\ddot{\eta}_{4} y_{0}-\ddot{\eta}_{5} x_{0}
\end{gathered}
$$

3.2. Limit State Function. Based on the acceleration components given by Eq. (3.2), a limit state function can be formulated as

$$
g=a_{0}-\sqrt{a_{y}^{2}+a_{z}^{2}}
$$

where $a_{0}$ is a threshold acceleration.

\section{Parallel System Analysis}

4.1. Stochastic Wave Elevation. The limit state function depends implicitly on the motion $\boldsymbol{\eta}$ of COG of the ship and, in general, the motion of the ship can be found by a time domain solution

$$
\boldsymbol{\eta}=\boldsymbol{\eta}(t)
$$

of the equations of motion. The stochastic excitation force is governed by the wave elevation $H(X, t)$, which for long-crested waves is a function of a global space coordinate $X$ in the direction of vessel propagation and time $t$. On the assumption that the stochastic wave elevation is a Gaussian process, $H(X, t)$ can be written as (Cramér and Leadbetter [2] may be consulted as a good general reference on stochastic processes)

$$
\begin{gathered}
H(X, t)=\sum_{n=1}^{N} A_{n} \cos \left(\omega_{n} t-k_{n} X+\epsilon_{n}\right) \\
=\sum_{n=1}^{N}\left[V_{n} c_{n}(X, t)+W_{n} d_{n}(X, t)\right]
\end{gathered}
$$


where $\omega_{n}$ and $k_{n}$ are discrete wave frequencies and wave numbers, respectively, related through the dispersion relation. With the sea states described by wave spectra $S(\omega)$, the deterministic coefficients $\left(c_{n}, d_{n}\right)$ are given by

$$
\begin{gathered}
c_{n}(X, t)=\sigma_{n} \cos \left(\omega_{n} t-k_{n} X\right) \\
d_{n}(X, t)=-\sigma_{n} \sin \left(\omega_{n} t-k_{n} X\right) \\
\sigma_{n}^{2}=S\left(\omega_{n}\right) \Delta \omega
\end{gathered}
$$

The variables $\left(V_{n}, W_{n}\right)$ are uncorrelated, standard normal distributed. They are related to the amplitudes $a_{n}$ and the phase lags $\epsilon_{n}$ through, e.g. Goda [5]

$$
\begin{gathered}
a_{n}=\sigma_{n} \sqrt{V_{n}^{2}+W_{n}^{2}} \\
\tan \epsilon_{n}=\frac{W_{n}}{V_{n}}
\end{gathered}
$$

The Pierson-Moskowitz (PM) spectrum is a simple and often reasonable approximation to ocean wave spectra of wind generated waves. The PM spectrum can be written as, e.g. Madsen et al. [14]

$$
S(\omega)=A \omega^{-5} \exp \left(-B \omega^{-4}\right)
$$

where

$$
A=\frac{H_{s}^{2}}{4 \pi}\left(\frac{2 \pi}{T_{z}}\right)^{4} \quad \text { and } \quad B=\frac{1}{\pi}\left(\frac{2 \pi}{T_{z}}\right)^{4}
$$

in which $H_{s}$ is the significant wave height and $T_{z}$ is the zero-upcrossing period.

From the complete description of the stochastic wave elevation, Eqs. (4.2)-(4.6), a time domain analysis yields the motion $\boldsymbol{\eta}(t)$ of the ship and, hence, the acceleration $a=\sqrt{a_{y}^{2}+a_{z}^{2}}$ can be calculated.

Long-crested waves can, in many cases, be used as a good approximation to describe the interaction between wave and ship and, in addition, long-crested waves often results in wave loads on the conservative side. It is therefore assumed here that the waves are long-crested. This assumption may easily be relaxed, but at the expense of larger computational times.

4.2. Uncertainty Modelling. The solution of Eq. (4.1) is conditioned on the time-invariant set of $\mathbf{W}=\left(V_{n}, W_{n}\right)$, so that each of the realisations $a(t \mid \mathbf{W})$ represents the acceleration of one possible wave field represented by an arbitrary set of $\mathbf{W}$. The ship motion depends directly on the significant wave height, cf. Eqs. (4.5)-(4.6) and, in addition, the ship motion implicitly depends on a number of operational parameters such as vessel speed $U$, relative wave heading $\chi$, metacentric height $G M$. Obviously, these dependencies will be reflected in the acceleration components as well. In real operational conditions none of the parameters are, however, exactly known; instead the parameters can be modelled as uncertain variables. Thus, it might be assumed that each of the parameters is described by a probability distribution function (pdf).

By letting all the random variables be incorporated into the vector $\mathbf{Y}$, the acceleration at time $t$ depends on the outcome of the vectors $\mathbf{W}, \mathbf{Y}$, that is

$$
a(t) \equiv a(t, \mathbf{W}, \mathbf{Y})
$$


which means that the limit state function must fulfill, cf. Eq. (3.3),

$$
g\left(a(t, \mathbf{W}, \mathbf{Y}), a_{0}\right)>0
$$

to avoid failure.

As specified by Bitner-Gregersen and Skjong [1], the uncertainty modelling should, in principle, consider the kind of uncertainty - aleatory (natural, inherent) and/or epistemic (knowledge) - in question for the individual parameters for the modelling to be complete. In the present paper, the actual modelling is, however, of less importance, since the objective concerns the development of a methodology to calculate outcrossing rates. In the numerical example which follows in a later section, only the epistemic uncertainties are considered in relation to the precision of the variables, without going into details about the origin of the random errors.

4.3. The Inner Loop - The Design Point. In accordance with Der Kiureghian [12] and e.g. Jensen and Capul [8], the realisation of the acceleration that exceeds a given threshold $a_{0}$ at time $t=t_{0}$ is sought for a given outcome of $\mathbf{Y}=\mathbf{y}$. Hence, the event of failure is formulated as a conditioned time-invariant reliability problem

$$
g\left(a\left(t=t_{0}, \mathbf{W} \mid \mathbf{Y}=\mathbf{y}\right), a_{0}\right)<0
$$

to which the solution is given by the design point $\mathbf{W}^{*}=\left(V_{n}^{*}, W_{n}^{*}\right)$, where the design point is the shortest distance from the origin to the limit state surface in the hyperspace of $\mathbf{W}$, e.g. Madsen et al. [14] and Ditlevsen and Madsen [3].

The solution, leading to the design point $\left(V_{n}^{*}, W_{n}^{*}\right)$, of this problem can be approximated by use of first order reliability methods (FORM), e.g. Madsen et al. [14] and Ditlevsen and Madsen [3]. The FORM analysis can be carried out by standard reliability codes, e.g. Proban developed at DNV [4]. Hence, $a\left(t=t_{0}, \mathbf{W} \mid \mathbf{Y}=\mathbf{y}\right)$ is calculated for a number of combinations of $\left(V_{n}, W_{n}\right)$ until the design point is reached. Then, based on the design point, a so-called critical wave episode, e.g. Jensen and Capul [8], can be determined by Eqs. (4.2) and (4.3) with $\left(V_{n}, W_{n}\right)=\left(V_{n}^{*}, W_{n}^{*}\right)$. This means that the critical wave episode is defined as the most probable wave episode leading to exceedance of the limit state.

It is important to emphasise that the processes considered are assumed to be stationary and ergodic. The only requirement to the time $t_{0}$ is therefore that the period $\left[0 ; t_{0}\right]$ is sufficiently long, so that the initial conditions at $t=0$ do not influence on the motion $\boldsymbol{\eta}\left(t_{0}\right)$. Usually, the memory of wave-structure interactions (such as ship responses) is less than 1-2 minutes which means that approximately $N=15-50$ discrete wave components of a typical wave spectrum should be used in order to avoid repetition in the wave system, cf. Jensen and Capul [8].

The reliability index $\beta_{F O R M}$ is defined as the distance from the origin to the design point $\left(V_{n}^{*}, W_{n}^{*}\right)$ on the limit state surface $g=0$ in the $2 N$-dimensional space of $\left(V_{n}, W_{n}\right)$. Hence, based on the 
design point, it can be shown that the outcrossing rate is given by, e.g. Jensen and Capul [8]

$$
\begin{gathered}
\nu^{+}\left(a_{0} \mid \mathbf{Y}=\mathbf{y}\right)=\frac{1}{2 \pi} \exp \left(-\frac{1}{2} \beta_{F O R M}^{2}\right) \sqrt{\sum_{n=1}^{N}\left(\alpha_{n}^{* 2}+\bar{\alpha}_{n}^{* 2}\right) \omega_{n}^{2}} \\
\left\{\alpha_{n}^{*}, \bar{\alpha}_{n}^{*}\right\}=\frac{\left\{V_{n}^{*}, W_{n}^{*}\right\}}{\beta_{F O R M}}
\end{gathered}
$$

Thus, the outcrossing rate may be obtained by a single FORM analysis. It is noteworthy that if a SORM analysis is performed, only the FORM reliability being the argument to the exponential function should be replaced by the SORM reliability index. The reason is that the $\alpha$-vector is the direction (i.e. the gradient vector) towards the design point which is the same in FORM and SORM analyses.

4.4. The Outer Loop - Integrating out Uncertainties. The outcrossing rate in Eq. (4.10) is calculated conditional on the outcome of the random vector $\mathbf{Y}$, which describes the uncertainties in sea state parameters, heading, speed, etc. In decision support systems it is the expected value of the mean outcrossing rate which is of interest, cf. Eqs. (2.1) and (2.2). The expected mean outcrossing rate is defined by

$$
\bar{\nu}(\zeta)=\int_{0}^{\infty}\left[1-F_{N^{+}}\left(\nu^{+}\right)\right] d \nu^{+}
$$

in which $F_{N^{+}}\left(\nu^{+}\right)$is the cumulative distribution function for the outcrossing rate $\nu^{+}$. For a specific target outcrossing $\nu_{\text {target }}$ it follows that

$$
\begin{gathered}
F_{N^{+}}\left(\nu_{\text {target }}\right)=P\left[N^{+}<\nu_{\text {target }}\right] \\
=\int_{N^{+}<\nu_{\text {target }}} f_{N^{+}}\left(\nu^{+}\right) d \nu^{+} \\
=\int_{\text {all } \mathbf{y}} \nu(\zeta \mid \mathbf{Y}=\mathbf{y}) f_{\mathbf{Y}}(\mathbf{y}) d \mathbf{y}
\end{gathered}
$$

where $f_{\mathbf{Y}}(\mathbf{y})$ is the joint density function of the random vector $\mathbf{Y}$, and it is noted that $\nu(\zeta \mid \mathbf{Y}=\mathbf{y})$ is given by the inner loop, cf. Eq. (4.10). The integral in Eq. (4.12) may be calculated by different means. A straightforward approach is to use Monte Carlo simulation. However, the main complexity in solving the integral is due to the cost of calculating the integrand for multiple outcomes of the random vector $\mathbf{Y}$. It is therefore suggested to use the parallel system analysis instead.

The term "parallel system analysis" stems from the fact that two systems are analysed in parallel; an outer loop 'circumscribing' an inner loop. To apply the parallel system approach it is necessary to formulate the analysis as a nested analysis. This requires the specification of two limit states. The first - and inner - limit state is defined by Eq. (4.9). This limit state returns the reliability index $\beta_{F O R M}$ of the event; calculated conditional on an arbitrary outcome of the $\mathbf{Y}$-vector. The reliability index of the inner analysis is made accessible to the outer limit state in the calculation of the probability of the event

$$
\nu^{+}-\nu_{\text {target }}<0
$$


which correspond to the evaluation of Eq. (4.12). Hence, by letting $\nu_{\text {target }}$ stepping through a (relevant) range of outcrossing rates, the cumulative distribution $F_{N^{+}}\left(\nu^{+}\right)$can be obtained and, finally, the expectation of the mean outcrossing rate may be estimated by Eq. (4.11). It is noted that the analysis is nested because each call to the outer analysis requires a full reliability analysis of the inner limit state Eq. (4.9).

As a final comment on the developed methodology - the parallel system analysis - it is important to note that the design point values from the inner loop, and thereby the whole solution, need to be used with some care. The reason is that nonphysical values can be obtained for the design point if the event considered is not properly mathematically formulated. In addition, some events are represented by failure surfaces that are not necessarily smooth, and this complicates the FORMassociated optimisation problem. These types of problems have not been observed in the present study, but an example of the latter problem can be seen in Nielsen [17] in relation to parametric rolling of a ship.

\section{The Equations of Motion}

The heave, $\eta_{3}$, and the pitch, $\eta_{5}$, motions can be described by linear theory and reasonable approximations for the RAOs may be obtained from the closed-form expressions by Jensen et al. [9]. Roll is a non-linear motion and based on the ROLLS procedure established by Kröger [13], Jensen and Pedersen [10] derived a simplified equation for the determination of the roll motion $\eta_{4}$,

$$
\ddot{\eta}_{4}+2 \beta_{1} \omega_{\eta_{4}} \dot{\eta}_{4}+\beta_{2} \dot{\eta}_{4}\left|\dot{\eta}_{4}\right|+\frac{\beta_{3} \dot{\eta}_{4}^{3}}{\omega_{\eta_{4}}}+\frac{\left(g-\ddot{\eta}_{3}\right) G Z\left(\eta_{4}\right)}{r_{x}^{2}}=\frac{M_{\eta_{4}}}{I_{x x}}
$$

in which $r_{x}$ is the roll radius of gyration, $M_{\eta_{4}}$ is the roll excitation moment, $I_{x x}$ is the mass moment of inertia about the longitudinal axis, and $\beta_{1}, \beta_{2}, \beta_{3}$ are damping coefficients. The roll frequency $\omega_{\eta_{4}}$ is given by the metacentric height $G M_{s w}$ in still water

$$
\omega_{\eta_{4}}=\frac{\sqrt{g G M_{s w}}}{r_{x}}
$$

In Eq. (5.1), the instantaneous value of the righting arm $G Z\left(\eta_{4}\right)$ is used. The calculation is based on the instantaneous wave height along the length of the vessel, so that the position of the wave crest is taken into account. The actual modelling can be found in Jensen and Pedersen [10].

The model in Eq. (5.1) is quite simplistic but it is still fully capable in producing results that illustrate phenomena such as parametric rolling, resonance excitation and forced rolling, see Jensen and Pedersen [10] and Jensen [7]. However, the model does not take surge into account, which means that broaching and dynamic rolling cannot be modelled. A refined version of the model including surge can be found in Jensen et al. [11].

The numerical solutions are obtained by embedding the time domain simulation routine, Eq. (5.1), combined with schemes for the heave and pitch accelerations into a standard FORM/SORM code (e.g. Proban, DNV [4]). The next section illustrates results obtained for the mean outcrossing rate related to the wave induced acceleration at a specified location, cf. Eqs. (3.2)-(3.3). 
TABLE 6.1. Main dimensions of the considered ship and position of calculated acceleration.

\begin{tabular}{lr}
\hline Length, $L_{\mathrm{pp}}$ & $284.7 \mathrm{~m}$ \\
Breadth, $B_{\mathrm{mld}}$ & $32.2 \mathrm{~m}$ \\
Draught, $T_{\text {mean }}$ & $10.5 \mathrm{~m}$ \\
Block coefficient, $C_{b}$ & 0.61 \\
Acc. in $(x, y, z)=$ & $(100,15,12) \mathrm{m}$ \\
\hline
\end{tabular}

\section{Example}

6.1. Considered Vessel. The example concentrates on a container vessel with main particulars given in Table 6.1. In the analysis, the wave induced acceleration is calculated at the position specified in the table; the position is given relative to centre of gravity of the vessel.

The $G Z$ curve of the vessel in still water is shown in Figure 6.1. For computational reasons the $G Z$ curves in still water and in waves are fitted by analytical expressions. These issues are treated in detail by Jensen and Pedersen [10] for the same vessel as studied here, and in the reference it is also shown how to deal with the time variation of the righting arm $G Z(t)$ in a stochastic seaway, using an equivalent wave procedure with some resemblance to that applied by Kröger [13].

6.2. Uncertainty Modelling of Random Variables. In addition to the operational parameters, all parameters entering Eq. (5.1) and those used for the curve-fitting of the $G Z$ curves can be modelled as random variables. In the present example, uncertainties are, however, only considered for significant wave height $H_{s}$, the zero-crossing wave period $T_{z}$, the relative wave heading $\chi$, the speed of the vessel $U$, and the metacentric height in still water $G M_{s w}$. Table 6.2 describes the assumed distributions and lists the parameters that need to be specified in the probabilistic analysis using Proban (DNV [4]). The actual values of the parameters follow below. The distributions and the corresponding parameter values in Table 6.2 are all based on rather intuitive presumptions and do not reflect any data as for justification. It is important to realise that the uncertainty modelling of the variables does not associate to the aleatory uncertainties (e.g. the long-term statistics) of the variables. Instead, the modelling relates to the epistemic uncertainties which are associated to

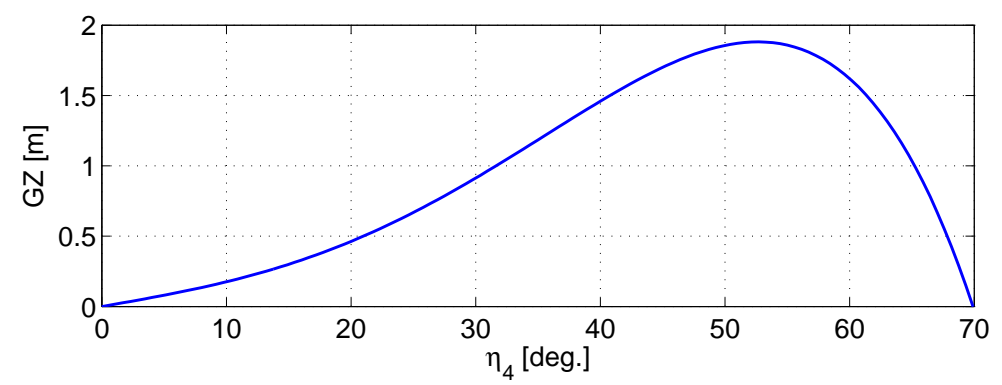

FIGURE 6.1. GZ curve in still water. 
TABLE 6.2. Uncertainty modelling of random variables. Mean: mean value; CoV: coefficient of variation; Low: lower limit; Lim: lower and upper limits.

\begin{tabular}{ccccc}
\hline Parameter & & Unit & Distribution & Values \\
\hline$H_{s}$ & Significant wave height & {$[\mathrm{m}]$} & Log-Normal & Mean-CoV-Low \\
$T_{z}$ & Zero-crossing period & {$[\mathrm{s}]$} & Log-Normal & Mean-CoV-Lov \\
$\chi$ & Relative wave heading & {$[\mathrm{deg} \cdot]$} & Trunc-Normal & Mean-CoV-Lim \\
$U$ & Speed of vessel & {$[\mathrm{m} / \mathrm{s}]$} & Log-Normal & Mean-CoV-Lov \\
$G M_{s w}$ & Metacentric height & {$[\mathrm{m}]$} & Normal & Mean-CoV \\
\hline
\end{tabular}

observation of the variables in a short-term sense. Moreover, it is noteworthy that no correlation are assumed between the parameters.

6.3. Specification of Input Data. Table 6.3 specifies the input data to the example which covers five cases, A, B, C, D, and E, representing different sets of parameters. In the example a PiersonMoskowitz wave spectrum, cf. Eq. (4.5), is assumed, and the spectrum is discretised into $N=25$ wave components. Figure 6.2 shows a plot of the spectrum, where the wave parameters are taken as the mean values listed in Table 6.3. In the calculations, the lower and upper cut-off frequencies are $\omega_{\text {low }}=0.15 \mathrm{rad} / \mathrm{s}$ and $\omega_{\text {upper }}=1.0 \mathrm{rad} / \mathrm{s}$, respectively, which covers the energy distribution reasonably well, cf. Figure 6.2. It is important to avoid repetition in the wave system and, therefore, the time domain solution cannot cover a period of more than approximately $180 \mathrm{~s}$ with the present discretisation and cut-off frequencies. In the computations the critical response, i.e. the wave induced acceleration, is sought at $t_{0}=100 \mathrm{~s}$, so that the memory effects in the system can be considered to be sufficiently small, there will be no problems related to repetition, and, still, the computational time is not maximum (as it would be for $t_{0}=180 \mathrm{~s}$ ). In all the analyses, the critical acceleration level is taken to be

$$
a_{0}=3.0 \mathrm{~m} / \mathrm{s}^{2}
$$

which is chosen on a somewhat arbitrary basis.

The probabilistic analysis is made by use of the software tool Proban, which is a general purpose probabilistic analysis program developed specifically to solve problems within reliability and probability contexts. In the present study, the optimisation problem related to the FORM calculations is handled by a non-linear programming method (NLPQL; e.g. DNV [4]) which solves a sequence of quadratic, linearly constrained sub problems.

In the evaluation of the limit state function a computational trick is applied to improve on the convergence, cf. DNV [4]. Thus, the limit state function, cf. Eq. (3.3), is replaced by

$$
g=\log t\left(a_{0}\right)-\log t\left(\sqrt{a_{y}^{2}+a_{z}^{2}}\right)
$$

where

$$
\operatorname{logt}(x)=\left\{\begin{array}{cc}
-1-\log (x) ; & x<-1 \\
x ; & -1 \leq x \leq 1 \\
1+\log (x) ; & 1<x
\end{array}\right.
$$


TABLE 6.3. Input data.

\begin{tabular}{|c|c|c|c|c|c|c|}
\hline \multirow[t]{2}{*}{ Case } & & & \multirow[t]{2}{*}{ Distribution } & \multicolumn{3}{|c|}{ Parameters } \\
\hline & & & & Mean & $\mathrm{CoV}$ & $\operatorname{Limit}(\mathrm{s})$ \\
\hline \multirow[t]{5}{*}{$\mathrm{A}$} & $H_{s}$ & {$[\mathrm{~m}]$} & Log-Normal & 9.0 & 0.20 & 1.0 \\
\hline & $T_{z}$ & {$[\mathrm{~s}]$} & Log-Normal & 11.0 & 0.15 & 3.0 \\
\hline & $\chi$ & [deg.] & Trunc-Normal & 135 & 0.20 & $110-160$ \\
\hline & $U$ & {$[\mathrm{~m} / \mathrm{s}]$} & Log-Normal & 9.0 & 0.10 & 3.0 \\
\hline & $G M_{s w}$ & {$[\mathrm{~m}]$} & Normal & 0.89 & 0.10 & 0.01 \\
\hline \multirow[t]{2}{*}{ B } & $\ldots$ & & as Case $A$ but & . & $\ldots$ & $\ldots$ \\
\hline & $\chi$ & [deg.] & Trunc-Normal & 150 & 0.20 & $125-175$ \\
\hline \multirow[t]{2}{*}{$\mathrm{C}$} & $\cdots$ & $\ldots$ & as Case $A$ but & $\ldots$ & $\ldots$ & $\ldots$ \\
\hline & $\chi$ & [deg.] & Trunc-Normal & 105 & 0.20 & 080-130 \\
\hline \multirow[t]{2}{*}{$\mathrm{D}$} & $\cdots$ & $\ldots$ & as Case $A$ but & $\ldots$ & $\cdots$ & $\ldots$ \\
\hline & $U$ & {$[\mathrm{~m} / \mathrm{s}]$} & Log-Normal & 6.0 & 0.10 & 3.0 \\
\hline \multirow[t]{2}{*}{$\mathrm{E}$} & $\ldots$ & $\ldots$ & as Case $A$ but & $\ldots$ & $\ldots$ & $\ldots$ \\
\hline & $H_{s}$ & {$[\mathrm{~m}]$} & Log-Normal & 12.0 & 0.20 & 1.0 \\
\hline
\end{tabular}

6.4. Results and Discussions. The main result by the parallel system analysis is basically given by the cumulative distribution of the outcrossing rate; the calculation procedure was described in Section 4. In regards to Case A, the cumulative distribution is shown in Figure 6.3 and, with reference to Eq. (4.11), the calculation of the corresponding expected value of the mean outcrossing rate yields $\bar{\nu}=2.50 \cdot 10^{-5} \mathrm{~s}^{-1}$ (see also Table 6.4 which will be introduced later). In comparison, Monte Carlo simulations (MCS) yield an expected value $\bar{\nu}=3.01 \cdot 10^{-5} \mathrm{~s}^{-1}$ with the associated expected coefficient of variation $\mathrm{CoV}=4.1 \%$, estimated from

$$
C o V=\frac{\sigma_{E v}}{\mu_{E v}} \approx \frac{1}{\sqrt{k \cdot \Delta t \cdot \nu}}
$$

where the variance of the zero-one variable $E v$ is given by $\operatorname{Var}(E v)=\sigma_{E v}^{2}=E\left[E v^{2}\right]-(E[E v])^{2}=$ $E[E v]-(E[E v])^{2}$ and with $E[E v]=\mu_{E v}=k \cdot \Delta t \cdot \nu$. Here $k \cdot \Delta t$ represents the total number of simulated time units (seconds). It should be noted that the target time of the parallel system analysis is $t_{0}=100 \mathrm{~s}$; however, in the specific case of Monte Carlo simulations of Case A, $k=$

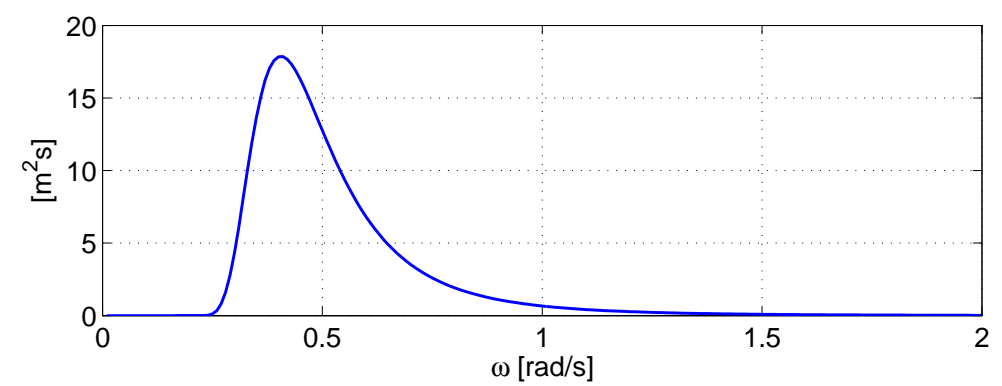

Figure 6.2. Pierson-Moskowitz wave spectrum. 
200, 000 time series simulations of length $t_{0}=150 \mathrm{~s}$ were carried out. Thus, the outcrossing count is determined from $100 \mathrm{~s}$ to $150 \mathrm{~s}$ (i.e. $\Delta t=50 \mathrm{~s}$ ) to minimise the influence of the initial conditions. As given in Table 6.4, the relative deviation between the results of parallel system analysis and MCS amounts to $-17 \%$ for Case A. Thus, the parallel system analysis approximates the expectation of the mean outcrossing rate with a reasonable accuracy.

As specified previously, the wave spectrum has been discretised into 25 wave components. It is, however, worth to mention that a refined discretisation, where 40 wave components are considered with the same cut-off frequencies, does not change the results of Case A; neither for the parallel system analysis, nor for the Monte Carlo simulations.

In the present analysis, five parameters are considered to be uncertain and, thus, these parameters are included in the parallel system analysis as random variables, cf. Table 6.3. In FORM calculations it is relatively straightforward to associate importance factors to the random variables. Figure 6.4 illustrates the relative importance of the random variables with respect to the analysis of Case A. In the plot, the base 10 logarithm is applied to $\nu$ and values lower than $1 \cdot 10^{-7} \mathrm{~s}^{-1}$ have been discarded in the plot. The discard is made in order to focus on periods, i.e. durations of time, and corresponding probability levels which are of the most relevance in decision support. From the figure it is observed that for low outcrossing rates the results are primarily influenced by the zero-crossing period $T_{z}$, which means that for outcrossing rates less than approximately $1 \cdot 10^{-5} \mathrm{~s}^{-1}$ (that is, events happening roughly once every 24 th hour of operation) it has the largest effect to change $T_{z}$ compared to changes of the other parameters. In reality, however, it makes little sense to talk about changing the zero-crossing period, since the parameter relates to the stochastic sea and, therefore, the ship master has no influence on the parameter. The importance of the zero-crossing period does, however, fade out and for outcrossing rates larger than approximately $5 \cdot 10^{-5} \mathrm{~s}^{-1}$ (that is, events happening roughly once every 5 th hour of operation) the results are mainly influenced - in relative terms - by the speed $U$ of the vessel and the significant wave height $H_{s}$. This means that, compared to the three other parameters, $T_{z}, \chi$ and $G M$, it has relatively the most effect on the mean outcrossing rate - under the adopted assumptions - to change, say, the speed, which is a parameter that is fully controllable by the ship master. The relative importance in changes of

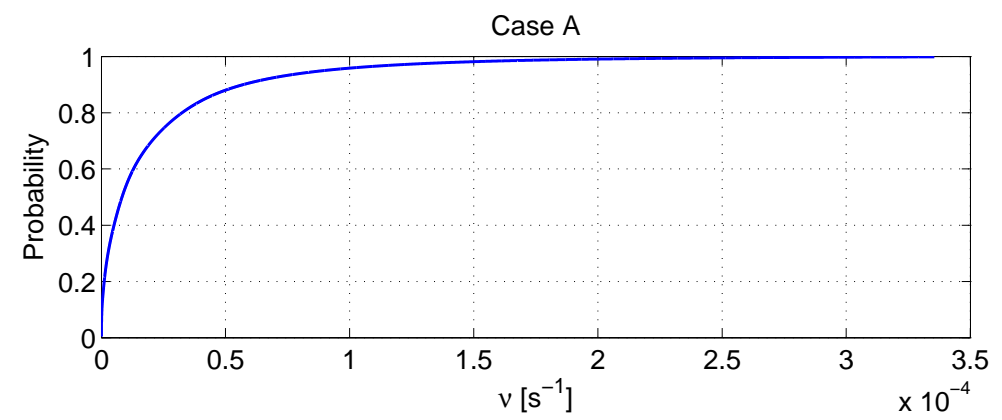

Figure 6.3. Case A; cumulative distribution of the mean outcrossing rate with $H_{s}$ $=9.0 \mathrm{~m}$ and $U=9.0 \mathrm{~m} / \mathrm{s}$. 
TABLE 6.4. The expected values of the mean outcrossing rates $\bar{\nu}\left[\mathrm{s}^{-1}\right]$ calculated by parallel system analysis and Monte Carlo simulations.

\begin{tabular}{cccccccc}
\hline Case & \multicolumn{2}{c}{ Par. sys. } & & & MCS & \multirow{2}{*}{ Dev. } \\
\cline { 2 - 3 } \cline { 5 - 6 } \cline { 5 - 6 } & Complete & $($ Inner $)$ & & Mean & CoV & Simul. & \\
\hline $\mathrm{A}$ & $2.50 \cdot 10^{-5}$ & $\left(8.73 \cdot 10^{-6}\right)$ & & $3.01 \cdot 10^{-5}$ & $4.1 \%$ & $2.0 \cdot 10^{5}$ & $-17 \%$ \\
$\mathrm{~B}$ & $1.69 \cdot 10^{-5}$ & $\left(3.46 \cdot 10^{-6}\right)$ & & $1.42 \cdot 10^{-5}$ & $5.9 \%$ & $2.0 \cdot 10^{5}$ & $19 \%$ \\
$\mathrm{C}$ & $4.42 \cdot 10^{-4}$ & $\left(4.02 \cdot 10^{-6}\right)$ & & $4.25 \cdot 10^{-4}$ & $1.5 \%$ & $1.0 \cdot 10^{5}$ & $4 \%$ \\
$\mathrm{D}$ & $3.43 \cdot 10^{-6}$ & $\left(2.52 \cdot 10^{-7}\right)$ & & $3.89 \cdot 10^{-6}$ & $4.1 \%$ & $15.0 \cdot 10^{5}$ & $-12 \%$ \\
$\mathrm{E}$ & $5.45 \cdot 10^{-4}$ & $\left(4.68 \cdot 10^{-4}\right)$ & & $10.8 \cdot 10^{-4}$ & $1.4 \%$ & $0.5 \cdot 10^{5}$ & $-50 \%$ \\
\hline
\end{tabular}

the heading and the metacentric height is almost constant around 5-10\% within most of the range of outcrossing rates. In the discussion on the relative importance of changing parameters it needs to be remembered that the limit(s), cf. Table 6.3 , associated to the parameters will influence the results.

The issues discussed above in regards to the relative importance of the environmental and operational parameters are to some extent reflected by the cumulative distributions of the other cases, B, C, D, and E, considered in Table 6.3. Thus, Figure 6.5 shows the cumulative distributions of the outcrossing rates in each of the four cases and, based on Eq. (4.11), the expected values of the mean outcrossing rates are obtained as given in Table 6.4 in the column 'Complete'. (The column 'Inner' typed in parentheses will be discussed later.) From the table it is seen that the expected mean outcrossing rate may be reduced about $32 \%$ to $1.69 \cdot 10^{-5} \mathrm{~s}^{-1}$ if the mean heading is changed from $\chi=135 \mathrm{deg}$. to $\chi=150 \mathrm{deg}$. as in Case B. On the other hand, with identical parameters but a mean heading $\chi=105 \mathrm{deg}$, the expectation of the mean outcrossing rate increases more than a factor of ten when Case $\mathrm{C}\left(\bar{\nu}=4.42 \cdot 10^{-4} \mathrm{~s}^{-1}\right)$ is compared to that of Case A. The reason is that the roll acceleration, in Case $\mathrm{C}$, contributes pronouncedly to the total acceleration. In relative terms, the largest effect on reducing the expected mean outcrossing rate is obtained by changing the speed. Thus, it is observed that a reduction to a mean speed $U=6.0 \mathrm{~m} / \mathrm{s}$, i.e. Case $\mathrm{D}$, leads to an expected value of the mean outcrossing rate $\bar{\nu}=3.43 \cdot 10^{-6} \mathrm{~s}^{-1}$; that is, approximately one tenth of that of Case A, which has all parameters identical but the speed. Finally, it is observed that for

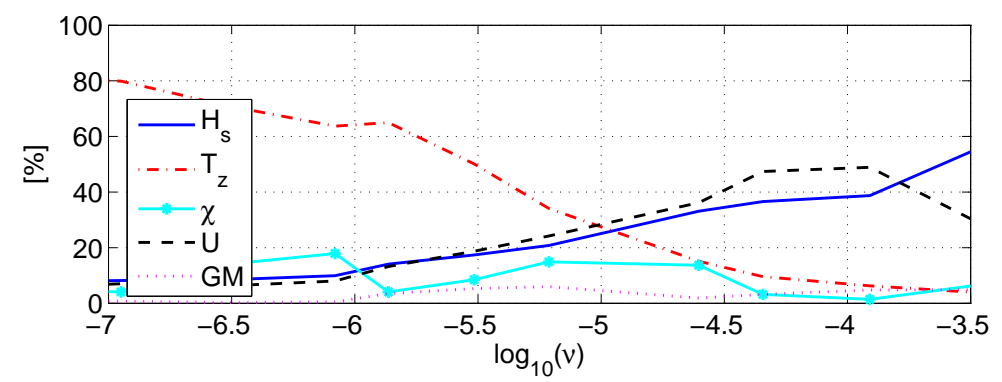

FigurE 6.4. Importance factors of Case A plotted versus the base 10 logarithm of $\nu$ with focus on a particular interval of $\nu$. 
identical parameters to that of Case A but with an increased significant wave height $\left(H_{s}=12 \mathrm{~m}\right)$, Case E, the expected mean outcrossing rate takes a value $\bar{\nu}=5.45 \cdot 10^{-4} \mathrm{~s}^{-1}$ which is more than ten times the value of Case A.

Monte Carlo simulations have also been carried out for all the cases and the results are included in Table 6.4. In each case, three values are given, that is, the mean value estimated from the simulations, the corresponding coefficient of variation (CoV) calculated by Eq. (6.4) and, finally, the number of simulations (Simul.) carried out. As evaluated by the coefficient of variation of the simulations, the results in terms of the mean values must be considered reliable and, hence, should make a reasonable background for comparison of the parallel system analysis. With respect to Cases A to D, the agreement between the results of the parallel system analysis and those of the Monte Carlo simulations is fair. Case E, on the other hand, exhibits a notable difference in the expected mean outcrossing rate of the two calculation procedures with the result of the parallel system analysis being $50 \%$ less relative to the result of simulations. It seems difficult to give an explanation for the relatively large deviation of Case E, so for now attention will be given to the fact that all results are of the same order of magnitude.

In the parallel system analysis, the outer loop integrates out uncertainties in the random variables $\mathbf{Y}$, so that the expected value of the mean outcrossing rate is obtained. If no uncertainties were present, so that all input parameters were deterministic, the expected mean outcrossing rate could therefore be obtained directly from the inner loop; that is, by Eq. (4.10). As an approximation, it is thus interesting to carry out an analysis, where all random variables of $\mathbf{Y}$ are replaced with their mean values as this greatly reduces the computational expenses. The results of this procedure are included in Table 6.4 in the column 'Inner' typed in parentheses. It is evident that, in general, this procedure approximates the complete and correct procedure badly. The somewhat fair agreement of Case $\mathrm{E}$ is obtained just by coincidence but one way to interpret this result is to say that for Case $\mathrm{E}$, the uncertainties have a low impact.
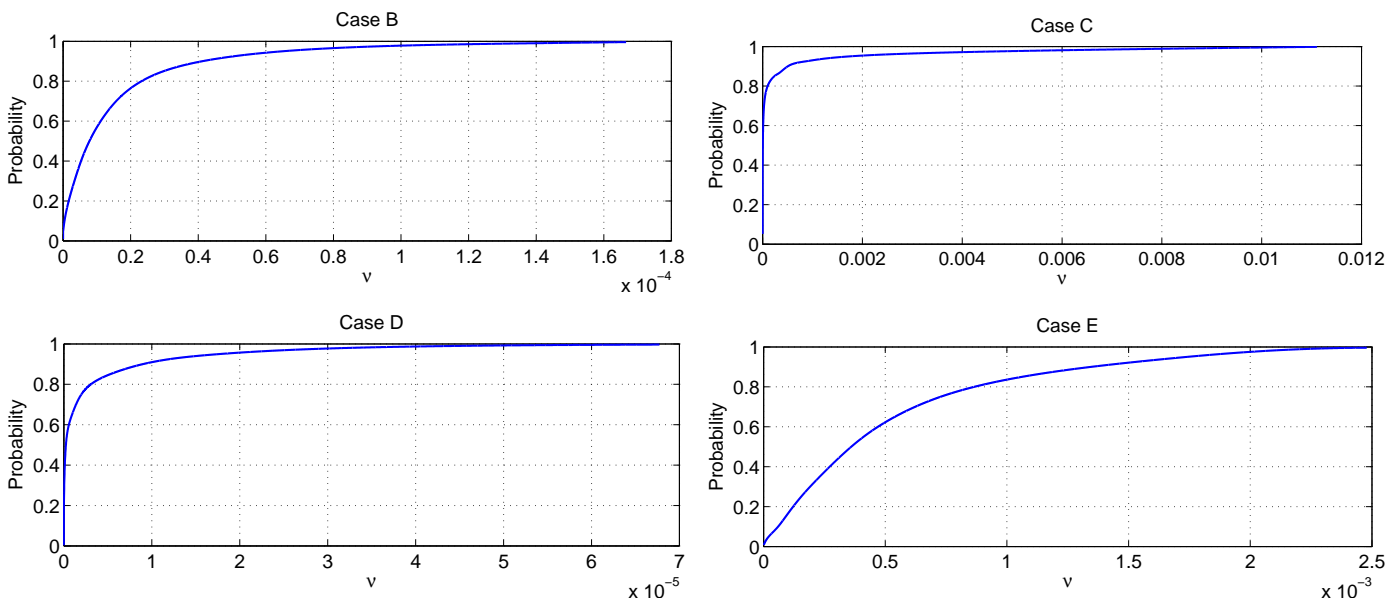

Figure 6.5. Cases B to E; cumulative distribution of $\nu\left(a_{0}=3.0 \mathrm{~m} / \mathrm{s}^{2}\right)$. Note the difference in the ranges/scales of $\nu$. 
One of the advantages of the parallel system analysis, cf. Table 2.1, is that the driving conditions, in terms of the most probable wave pattern leading to the specified response for the specific process can be easily extracted from the overall solution. The critical wave pattern is defined by the design point of W, cf. Eq. (4.9), and, in principle, the critical wave pattern can be used to reconstruct any type of response for the specific vessel and operational conditions. However, it needs to be emphasised that the actual wave pattern is the critical one with respect to one response - here the acceleration $a_{0}$ - which means that for other types of responses the critical wave pattern will most likely look differently. In Figure 6.6, time histories of the wave elevation at amidship and the corresponding most probable wave induced acceleration signal are shown for Case A. The specific realisations apply for an - arbitrarily chosen - outcrossing rate $\nu=2.49 \cdot 10^{-5} \mathrm{~s}^{-1}$ which is calculated at the global linearisation point $\left\{G M=0.893 \mathrm{~m}, U=9.30 \mathrm{~m} / \mathrm{s}, H_{s}=9.15 \mathrm{~m}, T_{z}=10.4 \mathrm{~m}, \chi=138 \mathrm{deg}\right.$. $\}$ of the FORM calculation. (The value of the outcrossing rate is by coincidence, approximately, equal to the expected mean outcrossing rate given in Table 6.4.) It is important to emphasise that the time variation of the acceleration is derived conditional on a wave induced acceleration of $a_{0}=3.0 \mathrm{~m} / \mathrm{s}$ at $t_{0}=100 \mathrm{~s}$. Moreover, it should be realised that the initial 40 seconds have been discarded in the plots, since this period of time represents, approximately, the duration in which the time variation is influenced by the initial conditions. Figure 6.6 is somewhat similar to plots shown and discussed by Jensen and Capul [8] and Jensen and Pedersen [10], although the references deal with other types of responses (deck sway of a jack-up unit and parametric roll, respectively) and do not take into account uncertainties in the dependent parameters. From Figure 6.6 it is noted how the acceleration gradually increases so that the acceleration hits $a_{0}=3.0 \mathrm{~m} / \mathrm{s}^{2}$ at $t_{0}=100 \mathrm{~s}$. It is seen that the maximum wave elevation amidship builds up and attains its maximum value about one quarter of a period before the maximum acceleration occurs. Moreover, it is noted that for the specific value of the heading, $\chi=138 \mathrm{deg}$, , the horizontal acceleration component plays a minor role compared to the vertical component. The latter phenomenon tends to even out the closer to beam sea the heading becomes. This is seen from Figure 6.7 that applies to Case C, where the relative wave heading has a mean value of $\chi=105 \mathrm{deg}$. The plot is based on the global linearisation point $\left\{G M=0.888 \mathrm{~m}, U=8.98 \mathrm{~m} / \mathrm{s}, H_{s}=9.04 \mathrm{~m}, T_{z}=10.1 \mathrm{~m}, \chi=096\right.$ deg. $\}$, so that $\nu=2.03 \cdot 10^{-4} \mathrm{~s}^{-1}$. Figure 6.7 also shows the time variation of the roll angle, and it is
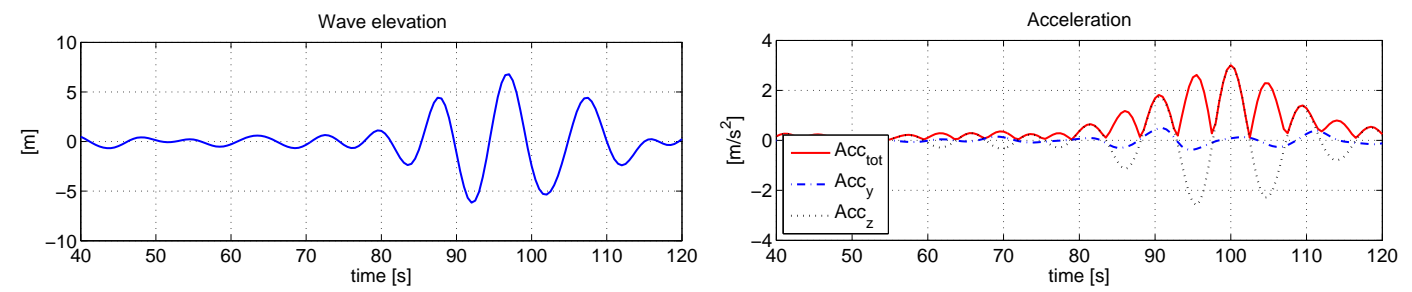

FIGURE 6.6. Time variation of critical wave elevation amidship and the corresponding wave induced acceleration for a specific outcrossing rate $(\nu=2.49$. $10^{-5} \mathrm{~s}^{-1}$ ) of Case $A$ with $G M=0.893 \mathrm{~m}, U=9.30 \mathrm{~m} / \mathrm{s}, H_{s}=9.15 \mathrm{~m}$, $T_{z}=10.4 \mathrm{~m}$, and $\chi=138 \mathrm{deg}$. 

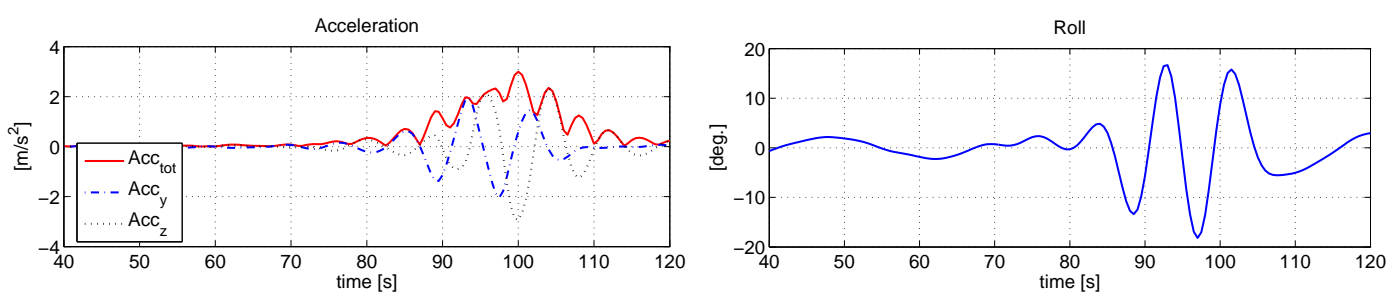

FigurE 6.7. Development in time of acceleration and roll angle for Case $C$; the outcrossing rate is $\nu=2.03 \cdot 10^{-4} \mathrm{~s}^{-1}$.

noted that the roll angle is in phase with the horizontal acceleration component, which also follows directly from Eq. (3.2).

The horizontal variation in space of the critical wave episode in the longitudinal direction of the ship is visualised in Figure 6.8 for two different outcrossing rates of Case A. Both profiles are conditioned on $a_{0}=3.0 \mathrm{~m} / \mathrm{s}^{2}$ at $t_{0}=100 \mathrm{~s}$, and both profiles are shown at exactly $t=t_{0}$. The ship propagates towards the positive direction of $x$ and, in the plots, the ship is indicated by a dashed box, so that the apparent wave length can be directly compared to the length of the ship. In regards to the wave development in space and time, the wave profile in the right plot of Figure $6.8\left(\nu=2.49 \cdot 10^{-5} \mathrm{~s}^{-1}\right)$, is, of course, seen to match the time variation of the wave elevation at amidship observed from Figure 6.6, which applies to the same outcrossing rate. From Figure 6.8 it is observed that the shapes of the space variation are similar in the two situations (i.e. left and right), although the actual values vary slightly; and this is the general picture (although not shown) for arbitrary outcrossing rates of Case A. It should be realised that the relatively long wave length, which appears from the figure, has to do with the fact that the vessel encounters the waves at an angle approximately equal to $\chi=135 \mathrm{deg}$. (specifically, $128 \mathrm{deg}$. and $138 \mathrm{deg}$. for the left and right plots, respectively). The real wave length can be obtained by multiplication with $\cos \chi$.

\section{Evaluation of the Procedure and Recommendations for Future Work}

The procedure of parallel system analysis is a useful tool for doing risk-based decision support since the method offers an efficient way to handle uncertainties of random variables when calculating mean outcrossing rates using FORM calculations. Some of the advantages of the procedure were
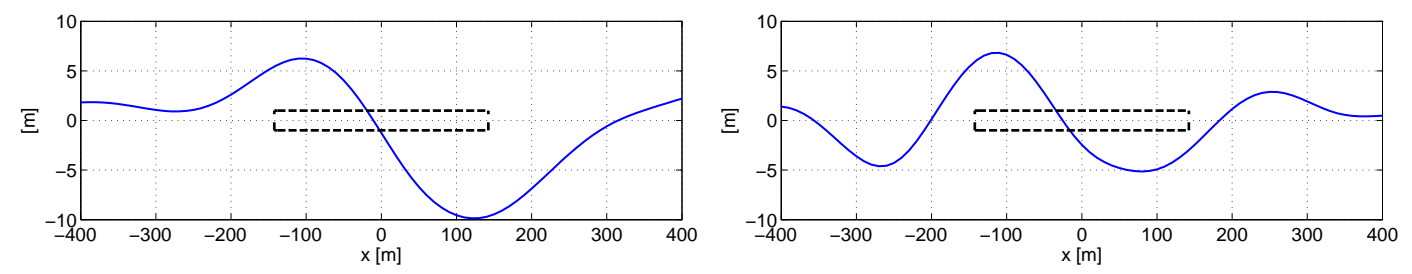

FIGURE 6.8. Horizontal variation in space of the critical wave episode for $\nu=2.06 \cdot 10^{-9} \mathrm{~s}^{-1}$ (left) and $\nu=2.49 \cdot 10^{-5} \mathrm{~s}^{-1}$ (right) of Case A. The amidship position is at $x=0 \mathrm{~m}$ as indicated by the dashed box. 
enlightened in the preceding example with respect to results and visualisations; e.g. possible provision of driving conditions in terms of a critical wave episode, and the calculation of importance factors. Furthermore, in comparison to brute force simulations the procedure of parallel system analysis might be considerably faster in the evaluation of the expected value of the mean outcrossing rate; in particular with respect to processes/events of rare occurrences. This was actually one of the main reasons for developing the procedure. However, it is important to note that the parallel system analysis has not proved as fast in the calculation time of the mean outcrossing rates as were initially expected. Specifically, the computational times (with a Pentium 2.0 GHz processor) were in the order of 4-8 hours for the calculation of the expected mean outcrossing rates for the cases considered in the preceding example. In comparison, the Monte Carlo simulations lasted for 3-50 hours in the specific cases depending on the probability level; i.e. the number of occurrences of the event. The calculation time of the parallel system analysis depends on the discretisation of the interval of outcrossing rates $\nu$ specified for the establishment of the cumulative distribution. Hence, the coarser discretisation of the interval, the faster the computation, so that if the relevant interval of outcrossing rates were known in advance, then it would be possible to reduce the calculation time. It would also be possible to reduce the computational time of the parallel system analysis by omitting the calculation of importance factors. Obviously, computational times in the order of hours are not acceptable for real-time onboard decision support systems, and therefore the parallel system analysis in its present state cannot be recommended for doing practical/operational risk-based decision support. In the future, work needs therefore to be carried out so that the computational speed of the parallel system analysis is increased.

The numerical example studied in the previous section dealt with only one type of response. Thus, it is necessary to extend the analysis to cover other types of responses in order to verify the procedure. Moreover, sensitivity studies need to be conducted on a higher level of details so that the procedure is checked for consistency; studies should be carried out with respect to e.g. number of wave components, the influence/importance of cut-off frequencies in the wave spectrum, the influence of length of simulation time $t_{0}$, the type of optimisation algorithm in regards to computational speed.

\section{Summary and Conclusions}

The parallel system analysis in its present form might be a step towards full risk-based decision support for ship operations under non-normal conditions such as heavy weather and high sea. The procedure is rather general and, by use of FORM/SORM calculations, it provides expected values of mean outcrossing rates even for very non-linear types of responses/limit states. All input parameters can be included in the analysis as random variables with associated statistical information and, in this way, the predictions/estimations should, theoretically, be the most correct. In addition, parallel system analysis offers the calculation of sensitivity factors, so that the relative importance of the input parameters can be evaluated. Furthermore, the driving conditions for the processes will be an immediate output from the analysis in terms of critical wave episodes. 
In the paper, a parallel system analysis was developed and the procedure was illustrated by an example which focussed on the wave induced acceleration at a specific location. The contributions to the acceleration were from heave, pitch and roll motions. Closed-form expression were utilised for the former two components whereas the roll motion was calculated by a non-linear equation, Jensen and Pedersen [8], somewhat similar to that of the ROLLS procedure, e.g. Kröger [13]. The response calculations were integrated with the probabilistic software Proban, DNV [4], which offers the combined use of response calculations and a standard FORM/SORM code. Based on the developed procedure and the illustrative example, it can be concluded that

- The parallel system analysis can produce results in terms of expected mean outcrossing rates that agree - within engineering accuracy - with corresponding results of Monte Carlo simulations.

- It is straightforward to obtain importance factors by sensitivity calculations from the parallel system analysis using FORM. This enables the evaluation of the relative importance of the different dependent parameters.

- Depending on the probability level the procedure of parallel system analysis is a much faster procedure for computing mean outcrossing rates compared to brute force simulations. However, the procedure did not prove as computational efficient as initially expected.

- Due to computational times in the order of hours, parallel system analysis in the present state should only be considered as a step towards full risk-based decision support.

\section{ACKNowledgement}

Fundamentals and initials of this work were carried out as part of WP3 within the ADOPT project under the Sixth Framework Programme of the European Commission, Contract No FP6-TST4-CT2005-516359, and the financial support is acknowledged.

\section{REFERENCES}

1. E.M. Bitner-Gregersen and R. Skjong, Concept for a risk based Navigation Decision Assistant, Marine Structures, In press.

2. H. Cramér and M.R. Leadbetter, Stationary and Related Stochastic Processes, Wiley, 1967.

3. O. Ditlevsen and H.O. Madsen, Structural Reliability Methods, Wiley, 1996, (The second edition from 2007 is available at the internet http://www.mek.dtu.dk/staff/od/book.htm).

4. DNV, Proban Theory, General Purpose Probabilistic Analysis Program, Det Norske Veritas, 2002.

5. Y. Goda, Random Seas and Design of Maritime Structures, Advanced Series on Ocean Engineering, vol. 15, World Scientific, 2000.

6. T. Iseki and D. Terada, Bayesian Estimation of Directional Wave Spectra for Ship Guidance Systems, International Journal of Offshore and Polar Engineering 12 (2002), 25-30.

7. J.J. Jensen, Efficient Estimation of Extreme Non-linear Roll Motions using the First-order Reliability Method (FORM), Journal of Marine Science and Technology 12 (2007), no. 4, 191-202.

8. J.J. Jensen and J. Capul, Extreme Response Predictions for Jack-up Units in Second Order Stochastic Waves by FORM, Probabilistic Engineering Mechanics 21 (2006), no. 4, 330-338.

9. J.J. Jensen, A.E. Mansour, and A.S. Olsen, Estimation of Ship Motions Using Closed-form Expressions, Ocean Engineering 31 (2004), 61-85. 
10. J.J. Jensen and P.T. Pedersen, Critcal wave episodes for assesment of parametric roll, Proceedings of IMDC'06, 2006, pp. 399-411.

11. J.J. Jensen, P.T. Pedersen, and J. Vidic-Perunovic, Estimation of Parametric Roll in a Stochastic Seaway, Proceedings of IUTAM Symposium on Fluid-Structure Interaction in Ecean Engineering (Hamburg, Germany), 2007.

12. A. Der Kiureghian, The Geometry of Random Vibrations and Solutions by FORM and SORM, Probabilistic Engineering Mechanics 15 (2000), 81-90.

13. H.-P. Kröger, Rollsimulation von Schiffen im Seegang, Schiffstechnik 33 (1986), 187-216.

14. H.O. Madsen, S. Krenk, and N. Lind, Methods of Structural Safety, Prentice Hall, New Jersey, USA, 1986.

15. J.K. Nielsen, N.H. Pedersen, J. Michelsen, U.D. Nielsen, J. Baatrup, J.J. Jensen, and E.S. Petersen, SeaSense Real-time Onboard Decision Support, Proceedings of WMTC2006 (London, UK), 2006.

16. U.D. Nielsen, Estimations of On-site Directional Wave Spectra from Measured Ship Responses, Marine Structures 19 (2006), no. 1, 33-69.

17. _ Calculating Outcrossing Rates used in Decision Support Systems for ships, Proc. of IMECE 2008 (Boston, MAS, USA), November 2008.

18. OCTOPUS Onboard, by the Dutch company Amarcon (http://www.amarcon.com).

19. Seaware EnRoute Live, by the Swedish company Seaware Onboard (http://www.seaware.se).

20. D. Spanos, A. Papanikolaou, and G. Papatzanakis, Risk-based Onboard Guidance to the Master for Avoiding Dangerous Seaways, 6th Osaka Colloquium on Seakeeping and Stability of Ships (Osaka, Japan), 2008.

21. J. Tellkamp, H. Günther, A. Papanikolaou, S. Krüger, K.-C. Ehrke, and J.K. Nielsen, ADOPT - Advanced Decision Support System for Ship Design, Operation and Training - An Overview, Proc. of COMPIT'08 (Liege, Belgium), April 2008, (http://www.anast.ulg.ac.be/COMPIT08/).

22. Vessel Optimization and Safety System, by the US company Ocean Systems Inc. (http://www.oceansystems.com). 